Revista Brasileira de Agricultura Irrigada v.9, no.4, p. 193 - 203, 2015

ISSN 1982-7679 (On-line)

Fortaleza, CE, INOVAGRI - http://www.inovagri.org.br

DOI: 10.7127/rbai.v9n400310

Protocolo 310.15 - 24/05/2015 Aprovado em 10/07/2015

\title{
SENSIBILIDADE DOS MICROTUBOS À OBSTRUÇÃO POR AGENTES FÍSICOS
}

\author{
Dinara Grasiela Alves ${ }^{1}$, Marinaldo Ferreira Pinto ${ }^{2}$, Bruno Molle $e^{3}$, Severine Tomas ${ }^{4}$, Nassim \\ Ait Mouheb ${ }^{4}$, Tarlei Arriel Botrel ${ }^{5}$
}

\begin{abstract}
RESUMO
Os microtubos são gotejadores de longo percurso feitos de polietileno e, recentemente, foi desenvolvido um novo sistema de funcionamento denominado de microtubo com múltiplas saídas (MMS) o qual consiste de um microtubo regulador de pressão ligado a um conector com disposição radial de onde derivam seis microtubos emissores. Os microtubos possuem elevada susceptibilidade ao entupimento, por causa de seu pequeno diâmetro. Objetivou-se com este trabalho avaliar a sensibilidade dos microtubos à obstrução por agentes físicos. Utilizou-se a metodologia proposta pelo Institut National de Recherche en Sciences et Technologies pour l'Environnement et l'Agriculture (IRSTEA) para avaliar a sensibilidade dos microtubos à obstrução. Além disso, foi utilizado o programa computacional ANSYS Fluent ${ }^{\circledR}$ para melhor visualização das áreas mais propícias à obstrução do MMS. Foram ensaiados microtubos com diâmetro de $1,074 \mathrm{~mm}$ e microtubos com múltiplas saídas funcionando sob pressão de entrada de $5 \mathrm{~m}$. O MMS é menos sensível à obstrução que os microtubos individuais com diâmetros internos de $1,074 \mathrm{~mm}$. O uso do programa ANSYS Fluent permitiu identificar as áreas críticas no conector que podem contribuir para a obstrução do emissor.
\end{abstract}

Palavras-chave: entupimento, emissor, inovação tecnológica, múltiplas saídas

\section{SENSIBILITY OF THE MICROTUBES TO OBSTRUCTION BY PHYSICAL AGENTS}

\footnotetext{
ABSTRACT

The microtube are emitters of small diameter and there has recently been developed a new type of microtube called of microtube with multiple outputs (MMO) and consists of a

${ }^{1}$ Pós-doutoranda, Departamento de Engenharia, Instituto de Tecnologia, UFRRJ, Seropédica - RJ. Email: dinara_alves@hotmail.com

${ }^{2}$ Doutor, Professor Adjunto, Departamento de Engenharia, Instituto de Tecnologia, UFRRJ, Seropédica - RJ. Email: mfpufrrj@yahoo.com.br

${ }^{3}$ Engenheiro, Institut national de recherche en sciences et technologies pour l'environnement et l'agriculture, Aix en Provence-França. E-mail: bruno.molle@ irstea.fr

${ }^{4}$ Doutor, Institut national de recherche en sciences et technologies pour l'environnement et l'agriculture, Montpellier-França. Email: severine.tomas@ irstea.fr; nassim.ait-mouheb@irstea.fr

${ }^{5}$ Doutor, Professor Titular, Departamento de Engenharia de Biossistemas, ESALQ, Piracicaba-São Paulo.

Email: tabotrel@usp.br
} 
pressure regulator microtube attached to a connector with radial arrangement from where are derived six emitters microtubes. The microtubes are highly susceptible to clogging because of its small diameter. The aim of this study was to evaluate the sensitivity of the microtubes to obstruction by physical agents. We used the methodology proposed by Institut National de Recherche en Sciences et Technologies pour l'Environnement et l'Agriculture (IRSTEA) to evaluate the sensitivity of the microtubes to obstruction. In addition, we used the computational program ANSYS Fluent for better visualization of areas more conducive to obstruction of the MMO. Microtubes were assayed with a diameter of $1.074 \mathrm{~mm}$ and microtubes with multiple outlets operating with inlet pressure of $5 \mathrm{~m}$. The MMO is less sensitive to clogging than the individual microtubes with internal diameters of $1.074 \mathrm{~mm}$. The use of ANSYS Fluent program identified critical areas in the connector that can contribute to the obstruction of the emitter.

Keywords: obstruction, emitter, technological innovation, multiple outputs

\section{INTRODUÇÃO}

Os microtubos são gotejadores de longo percurso feitos de polietileno de pequeno diâmetro, de baixo custo, fácil instalação e manutenção, destacando-se como um importante instrumento para a elevação do nível tecnológico e competitividade de pequenos irrigantes (SOUZA, 2005; ALVES, 2010). Nesse sentido, recentemente foi desenvolvido por Alves (2010) um novo tipo de microtubo denominado de microtubo com múltiplas saídas (MMS), o qual ainda é inexistente no mercado e consiste de um microtubo regulador de pressão (MRP) ligado a um conector com disposição radial de onde derivam seis microtubos emissores (ME).

Esses gotejadores, quando dimensionados corretamente, possibilitam elevada uniformidade de distribuição de água. Contudo, os mesmos apresentam algumas desvantagens como: alta sensibilidade à variação de temperatura e pressão quando trabalha em regime laminar (FRIZZONE et al., 2012) e elevada susceptibilidade ao entupimento (SOUZA; BOTREL 2004; ALMEIDA; BOTREL; SMITH, 2009).

Em ocasiões em que não são executadas medidas preventivas, a obstrução é considerada como o principal problema em microirrigação, podendo ocasionar danos em todo o processo produtivo, por afetar a taxa de aplicação e a uniformidade de distribuição da água, elevando os custos de manutenção, de reposição de peças, de recuperação e com inspeções (FRIZZONE et al., 2012).

A obstrução dos emissores pode ocorrer por agentes físicos, químicos e/ou biológicos. A determinação da causa da obstrução pode ser complexa pelo fato de ocorrer vários agentes na água interagindo entre si, agravando o problema, sobretudo se for considerada a natureza dinâmica dos indicadores de qualidade de muitas águas (RAVINA et al., 1992).

Existem vários trabalhos sobre obstrução de emissores (TAJRISHY et al., 1994; KREIJ; VAN DER BURG; RUNIA, 2003; DURANROS et al., 2009; PUIG-BARGUÉS et al., 2010; LI; LI; ZHANG, 2012; SILVA et al., 2012) em que a maioria usam água residuária para avaliar a susceptibilidade de diferentes gotejadores à obstrução ou avaliam alternativas para evitar a obstrução. Almeida et al. (2013), avaliaram a susceptibilidade de microtubos ao entupimento sob fertirrigação e obteve grau máximo de entupimento de aproximadamente 50\%. Dentre as diferentes causas de obstrução, destaca-se o entupimento por causa física que pode ocorrer devido à presença de sólidos em suspensão no interior da tubulação. Estudos realizados por Gilbert et al. (1981) verificaram que a principal causa de entupimento e consequente redução da vazão dos emissores foi ocasionada por obstrução física, apresentando $55 \%$ das ocorrências.

Com os avanços tecnológicos é possível a utilização de métodos computacionais que permitam a visualização de áreas, no interior do 
emissor, mais propícias à ocorrência de entupimento. O programa ANSYS Fluent que utiliza o modelo Computacional de Dinâmica dos Fluidos (CFD) é uma ferramenta de simulação bastante usada para a previsão, visualização e investigação do movimento da água em gotejadores. Contudo, o valor da licença para utilizar essa ferramenta é bastante elevado. Wei et al. (2006, 2008), Gamri et al.

\section{MATERIAL E MÉTODOS}

\section{Condução do Experimento}

A condução dessa pesquisa dividiu-se em duas etapas: a primeira consistiu na determinação do diâmetro interno e do comprimento dos microtubos que foi realizado no Laboratório de Hidráulica do Departamento de Engenharia de Biossistemas da Escola Superior de Agricultura "Luiz de Queiroz"/USP, situada no município de Piracicaba - SP; a segunda consistiu na modelagem do escoamento d'água no interior do microtubo com múltiplas saídas, e, na avaliação da sensibilidade dos microtubos à obstrução por agentes físicos, que foram realizados no Laboratoire d'Essais et de Recherche des Matériels d'Irrigation pertencente ao Institut National de Recherche en Sciences et Technologies pour l'Environnement et l'Agriculture (LERMI/IRSTEA, Aix en Provence, Montpellier, França). Ressalta-se que, em todos os ensaios a temperatura da água foi mensurada a fim de obter o valor da viscosidade cinemática e o valor de sua massa específica.

\section{Descrição do microtubo com múltiplas saídas (MMS)}

O emissor consiste de um microtubo regulador de pressão (MRP) ligado a um conector com disposição radial de onde derivam seis microtubos emissores (ME) (Figura 1). Esse conector pode ser visualizado em maiores detalhes na Figura 2. Ressalta-se que, o MRP foi denominado deste modo pelo fato de seu comprimento poder ser ajustado de acordo com a pressão no ponto de inserção do MRP na linha
(2014) e Wu et al. (2013) analisaram as características do escoamento d’água em emissores de sistema de microirrigação, referindo-se principalmente a susceptibilidade dos gotejadores a obstrução, que é um dos principais problemas desse sistema.

Diante do exposto, o objetivo deste trabalho foi avaliar a sensibilidade dos microtubos à obstrução por agentes físicos.

lateral de forma a manter constante a pressão na parte central do conector.

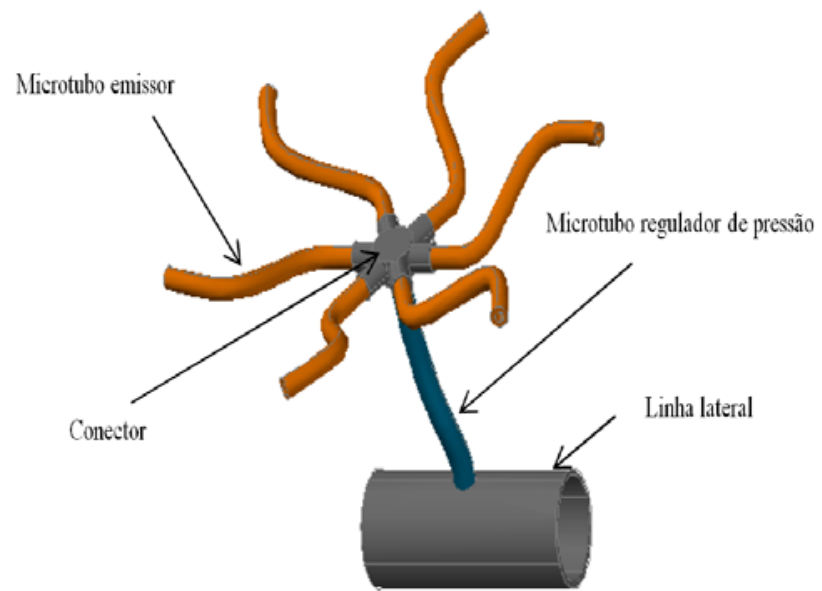

Figura 1. Esquema do microtubo com múltiplas saídas

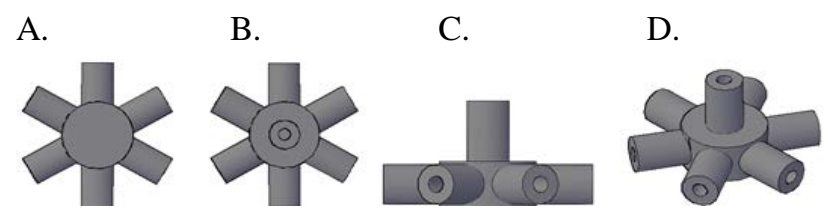

Figura 2. Conector: A - vista inferior; B - vista superior; C - vista lateral; D - vista em perspectiva

\section{Determinação do diâmetro interno dos microtubos}

Antes de serem realizados os testes com o emissor proposto, necessitou-se determinar o diâmetro real dos microtubos, pois, qualquer variação no diâmetro poderia ocasionar grande alteração no valor da vazão prevista no projeto.

Foram avaliados microtubos com diâmetro nominal de 0,8 e 1,0 mm, fabricados pela empresa Plasnova Louveira Ind. Com. Ltda. A determinação do diâmetro interno do microtubo foi realizada por meio de medidas hidrodinâmicas em laboratório, sob regime laminar, conforme metodologia proposta por 
Almeida e Botrel (2010). Para isso, foram utilizados microtubos com comprimento de $5 \mathrm{~m}$, submetidos a uma pressão constante de 12,4 $\mathrm{kPa}$. Os diâmetros internos dos microtubos foram calculados por meio de dados obtidos nos ensaios, aplicados na equação (1) proposta por Almeida e Botrel (2010).

$$
\mathrm{D}=\left(\frac{0,08262647 \mathrm{Q}^{2}+4,153269 \mu \mathrm{LQ}}{\mathrm{Z}}\right)^{0,25}
$$

em que:

D - diâmetro interno do microtubo, m;

$\mu$ - viscosidade cinemática da água, $\mathrm{m}^{2} \mathrm{~s}^{-1}$;

$\mathrm{L}$ - comprimento do microtubo, m;

$\mathrm{Q}$ - vazão no microtubo, $\mathrm{m}^{3} \mathrm{~s}^{-1}$; e

$\mathrm{Z}$ - carga de posição, $\mathrm{m}$.

Os diâmetros reais obtidos foram de 0,888 mm e 1,074 mm para os diâmetros nominais de 0,8 e 1,0 mm, respectivamente. O cálculo do comprimento dos MRP e ME, foi realizado com base em uma planilha eletrônica, utilizando para isso as seguintes variáveis independentes: pressão, diâmetro e vazão. Utilizou-se o modelo desenvolvido por Alves et al. (2012) para o dimensionamento dos microtubos. Admitiramse os seguintes valores no projeto: vazão de $1 \mathrm{~L}$ $\mathrm{h}^{-1}$ para o ME e de $6 \mathrm{~L} \mathrm{~h}^{-1}$ para MRP, viscosidade de $1,01 \times 10^{-6} \mathrm{~m}^{2} \mathrm{~s}^{-1}$ para uma temperatura de $23{ }^{\circ} \mathrm{C}$, pressão de alimentação de $49,05 \mathrm{kPa}$ e pressão na parte central do conector de $10,79 \mathrm{kPa}$.

\section{Simulação do escoamento d’água no microtubo com múltiplas saídas utilizando o software ANSYS Fluent}

Realizaram-se simulações numéricas utilizando o programa ANSYS Fluent ${ }^{\circledR}$ versão 14.5 que utiliza a interface Workbench. As simulações foram realizadas em três etapas: criação da geometria, criação da malha e modelagem. Utilizou-se a interface Design Modeler do ANSYS Workbench para criação da geometria que consiste em desenhar o trajeto de escoamento d'água no interior do microtubo com múltiplas saídas. Utilizou-se a interface Meshing do ANSYS Workbench para geração da malha que permite a solução numérica das equações de escoamento. Após a geração da malha, realizou-se o seu refinamento. Esse procedimento consiste em reduzir o tamanho máximo da malha na região desejada (Figura 3).

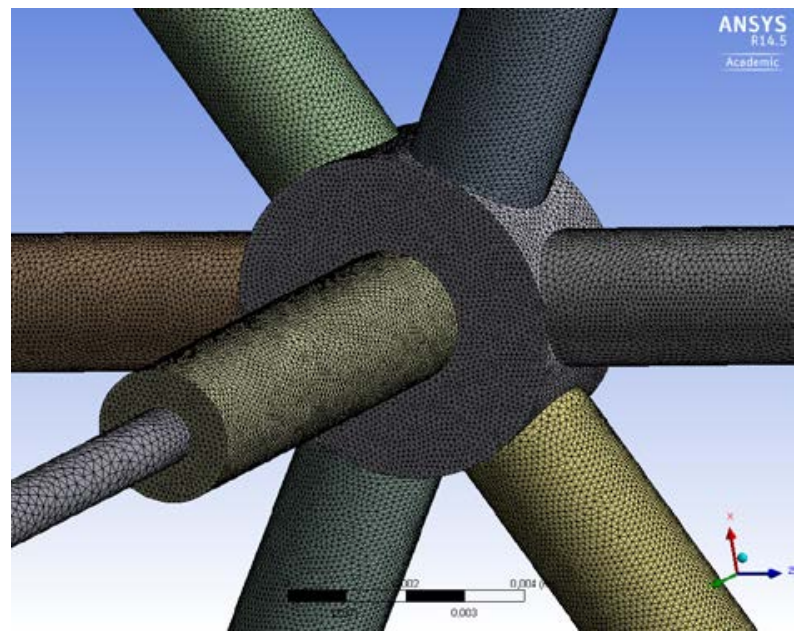

Figura 3. Malha do tipo tetraédrica no conector do microtubo com múltiplas saídas com tamanho máximo de $1,5 \times 10^{-4} \mathrm{~m}$

O regime de escoamento foi determinado em função do número de Reynolds (Re) de acordo com Porto (2006): laminar $(\operatorname{Re}<2300)$; transição ( $2300<\operatorname{Re}<4000)$; e turbulento (Re $>$ 4000). Utilizou-se o modelo k- $\varepsilon$ Standard, pois foi o que mais se aproximou dos resultados experimentais. Ressalta-se que $\mathrm{k}$ significa campo de energia cinética turbulenta $\left(\mathrm{m}^{2} \mathrm{~s}^{2}\right)$ e $\varepsilon$ é a taxa de dissipação de energia $\left(\mathrm{m}^{2} \mathrm{~s}^{3}\right)$. Realizou-se dois cortes ao longo do microtubo com múltiplas saídas, um no sentido longitudinal e outro no sentido transversal, para visualização das áreas propícias a ocorrência de obstrução por meio do campo de velocidade.

\section{Sensibilidade dos microtubos à obstrução por agentes físicos}

Utilizou-se a metodologia proposta pelo IRSTEA (2013) para avaliar a sensibilidade dos microtubos à obstrução por agentes físicos. Foram ensaiados três microtubos com comprimento de 0,410 m e diâmetro de 1,074 $\mathrm{mm}$ e três MMS (seis microtubos com comprimento de 0,584 m e diâmetro de 0,888 $\mathrm{mm}$ conectados a um microtubo com comprimento de 0,410 m e diâmetro de 1,074 $\mathrm{mm}$ ) funcionando sob pressão de entrada de 5 $\mathrm{m}$, a qual foi monitorada por um manômetro 
analógico. Antes de iniciar os testes de obstrução, os microtubos foram ensaiados com água limpa para obter a vazão nos emissores.

$O$ ensaio de sensibilidade à obstrução foi realizado em quatro fases com duração de uma semana cada. O tempo de funcionamento do sistema foi de oito horas por dia e cinco dias por semana, com exceção da primeira semana em que os ensaios foram realizados em apenas três dias. Na primeira semana foi acrescentada areia com diâmetro entre $0-80 \mu \mathrm{m}$ em um tanque de 100 litros de água filtrada; na segunda semana, adicionou-se a esta solução, areia com diâmetro entre 80-100 $\mu \mathrm{m}$; na terceira semana, adicionouse areia com diâmetro entre 100-200 ㅆm; e, na quarta semana foi inserida areia com diâmetro entre 200-500 $\mu \mathrm{m}$. Em cada etapa a concentração de areia acrescentada foi de 125 $\mathrm{mg} \mathrm{L}^{-1}$. Deste modo, no início da quarta semana, a concentração total de areia adicionada ao tanque foi de $500 \mathrm{mg} \mathrm{L}^{-1}$.

\section{RESULTADOS E DISCUSSÃO}

\section{Campo de velocidade}

Na Figura 4 pode-se observar o campo de velocidade $\left(\mathrm{m} \mathrm{s}^{-1}\right)$ obtido pelo modelo $\mathrm{k}-\varepsilon$. A velocidade máxima é verificada no MRP e na entrada do conector. Velocidade próxima à zero é observada na parede do conector e na zona de recirculação (região do conector onde a vazão é direcionada ao ME). Quanto menor a velocidade da água no interior do emissor, maior é a chance de deposição e acumulação de materiais presentes na água e, consequentemente, maior é a possibilidade de entupimento dos emissores. Dessa forma, verifica-se que a área do emissor proposto com maior risco à obstrução está presente nas saídas do conector. Áreas com valores de velocidade próximos ou igual a zero devem ser evitadas, sendo necessária a realização de adequações na arquitetura do conector para minimizar as chances de entupimento do emissor proposto.

No entanto, quanto maior a energia cinética maior será as colisões entre as partículas presentes na água, o que pode causar a aglomeração de pequenas partículas,
A solução foi homogeneizada por meio de um agitador mecânico e em seguida, foi bombeada para os microtubos. Em todos os ensaios a vazão foi medida por meio da coleta de água durante $5 \mathrm{~min}$ em recipientes de peso conhecido. A coleta era realizada de 3 a 4 vezes por dia e a temperatura era juntamente mensurada. A vazão foi determinada por meio da razão entre o volume de água coletado e tempo de coleta. Após finalizado o ensaio e desmontado o experimento, cortou-se as extremidades dos microtubos (entrada e saída) em tamanhos de $2 \mathrm{~mm}$ para serem observadas no microscópio. Utilizou-se um microscópio da marca Motic modelo SMZ-168 com câmera de 2 M Pixel. As imagens capturadas pela câmera foram utilizadas para visualização dos microtubos e dos conectores por meio de um programa computacional Motic Images Plus versão

2

ML.

transformando-se em partículas maiores, ocasionando a obstrução do emissor. Para Wei et al. (2006) o aumento da energia cinética afeta diretamente a dissipação de energia e a velocidade e consequentemente, a vazão do emissor. Provavelmente, o alargamento de seção e a mudança brusca de direção do fluxo da água causam alteração na velocidade da água no interior do emissor, principalmente na região próxima à entrada do conector e na região em que a água é direcionada para os microtubos emissores. Nessas regiões ocorrem as maiores taxas de perda localizada de carga. Resultados semelhantes foi verificado no trabalho de Wei et al. (2006).

Segundo Azevedo Netto (1998) a perda localizada de carga pode ser desprezada nas tubulações longas cujo comprimento exceda cerca de 40000 vezes o diâmetro. São ainda desprezíveis nas canalizações em que a velocidade é baixa e o número de peças especiais não é grande. No entanto, isto não se enquadra ao emissor proposto, pois, o comprimento do microtubo regulador de pressão é pequeno, além disso, no conector derivam seis emissores. O MRP pode ocasionar perdas localizada de carga decorrentes da inserção de 
parte de seu corpo no interior da linha lateral e no conector, funcionando como um ponto de formação, ou acréscimo, de turbulência. Aliado a isso, a inserção dos microtubos emissores no conector também pode contribuir para essas perdas.
A.

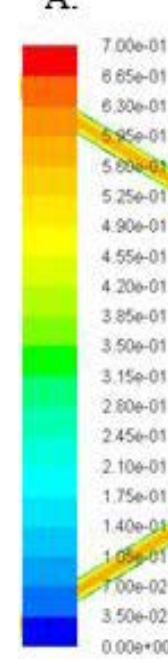

B.

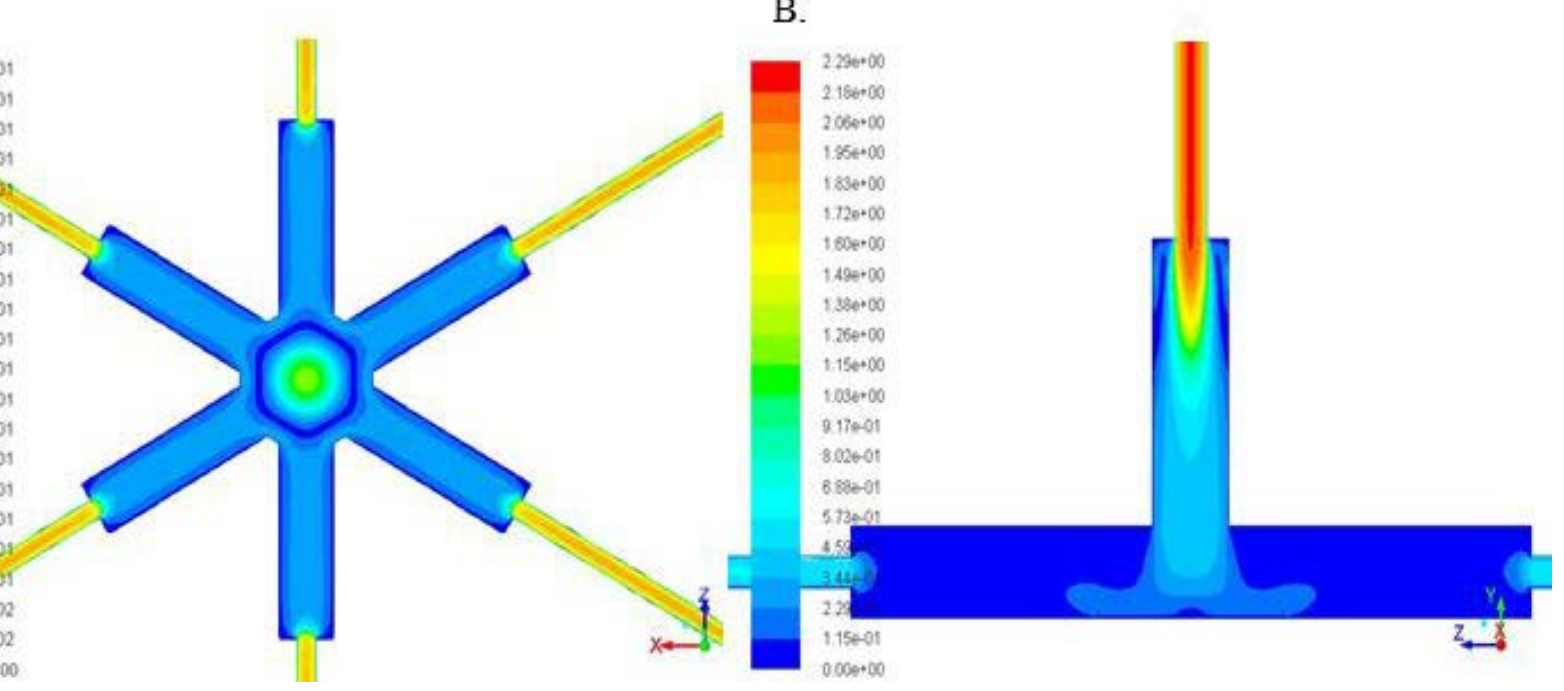

Figura 4. Campo de velocidade $\left(\mathrm{m} \mathrm{s}^{-1}\right)$ obtido pelo modelo k-e. A - plano horizontal; B - plano vertical

\section{Sensibilidade dos microtubos à obstrução por agentes físicos}

Os resultados dos ensaios realizados nos microtubos utilizando água limpa podem ser visualizados na Tabela 1.

Tabela 1. Vazão em cada microtubo e vazão média do microtubo (M) e microtubo com múltiplas saídas (MMS) em ensaio realizado com água limpa

\begin{tabular}{ccc}
\hline Microtubos & $\begin{array}{c}\text { Vazão } \\
\left(\mathrm{L} \mathrm{h}^{-1}\right)\end{array}$ & $\begin{array}{c}\text { Vazão média } \\
\left(\mathrm{L} \mathrm{h}^{-1}\right)\end{array}$ \\
\hline M1 & 7,91 & \\
M2 & 7,87 & 7,87 \\
M3 & 7,84 & \\
\hline MMS1 & 6,61 & \\
MMS2 & 6,80 & 6,68 \\
MMS3 & 6,64 & \\
\hline
\end{tabular}

Observa-se que a vazão obtida no ensaio relacionado ao MMS é um pouco superior à vazão definida no projeto. Isso provavelmente pode ter ocorrido devido à temperatura utilizada nos ensaios ter sido diferente da temperatura utilizada no projeto. Além disso, pequenas diferenças nos valores dos diâmetros dos microtubos também podem ter contribuído para isso.

\section{Microtubos}

O potencial de entupimento dos emissores é diferenciado em função de características como qualidade da água, diâmetro de passagem, velocidade do fluxo e das características físicas construtivas. Nesse sentido, os microtubos analisados neste trabalho possuem média sensibilidade à obstrução segundo a classificação proposta por Pizarro Cabello (1996) em função do diâmetro interno do emissor.

Na primeira semana os microtubos não obstruíram. Na segunda semana do ensaio apenas o microtubo 1 (M1) ficou obstruído cuja a vazão foi reduzida em 48,05\% após 63 horas de funcionamento do sistema. Na terceira semana os microtubos funcionaram regularmente com exceção do M1 que ficou totalmente obstruído. Na última etapa apenas o M2 funcionou normalmente até o final do 
ensaio, ou seja, se o sistema funcionasse por um período maior ele provavelmente seria obstruído. Logo, não é recomendável utilizar microtubos com diâmetro igual ou inferior a
$1,074 \mathrm{~mm}$ em solução que contenha areia com diâmetro superior a $100 \mu \mathrm{m}$ e concentração de areia igual ou superior a 250 mg $\quad \mathrm{L}^{-1} \quad$ (Figura

$5)$.

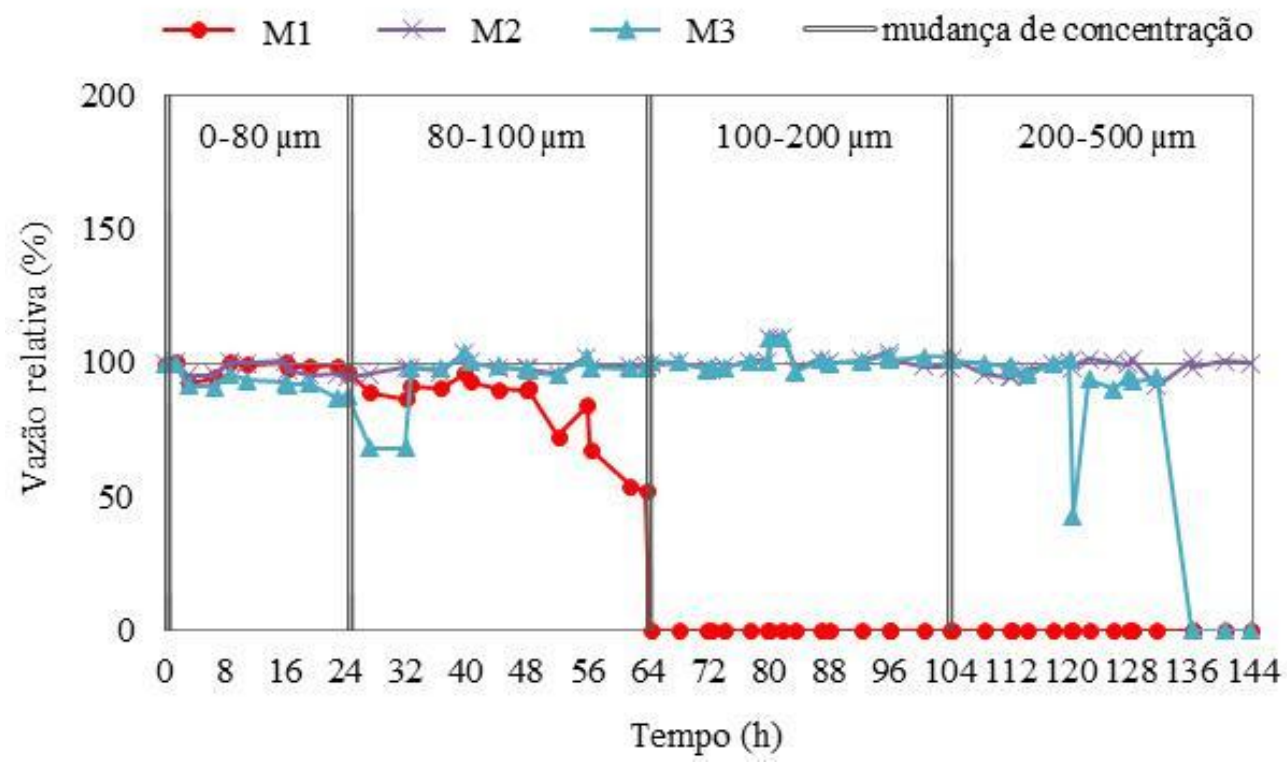

Figura 5. Vazão relativa dos microtubos reguladores de pressão (MRP) ao longo do tempo, com o aumento de concentração e diâmetro de partículas de areia na verificação da sensibilidade à obstrução

Ao analisar as extremidades desses microtubos, observou-se a presença de areia e de outros materiais que não puderam ser identificados (Figura 6). Supostamente o material fibroso visível na Figura 6A seja proveniente do filtro utilizado neste ensaio cujo elemento filtrante era de cordão de algodão.

A.

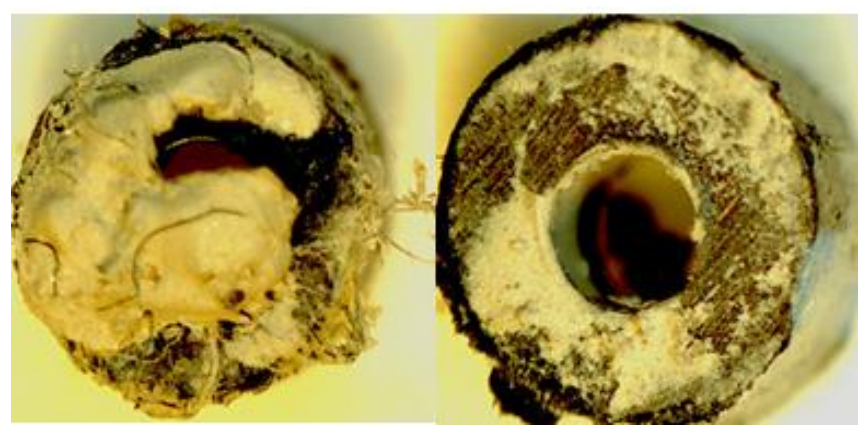

Figura 6. Microtubo obstruído. A - entrada do microtubo regulador de pressão; B - saída do microtubo regulador de pressão

\section{Microtubos com múltiplas saídas}

Observa-se que os MMS funcionaram regularmente nas três primeiras semanas de ensaio, ao contrário dos ME e MRP que apresentaram algum problema de obstrução. Isso pode ter acontecido pelo fato das partículas de areia ficarem acumuladas no interior do conector ao invés de passar pelos microtubos emissores. No final da quarta semana o microtubo com múltiplas saídas 2 (MMS2) estava completamente obstruído e os MMS1 e MMS3 estavam com a vazão reduzida em 58,45 e 61,69\% respectivamente (Figura 7). Logo, não é recomendável utilizar microtubos com múltiplas saídas (com a especificação utilizada no ensaio) em água que contenha areia com diâmetro superior a $200 \mu \mathrm{m}$ e concentração de areia igual ou superior a $375 \mathrm{mg} \mathrm{L}^{-1}$. 


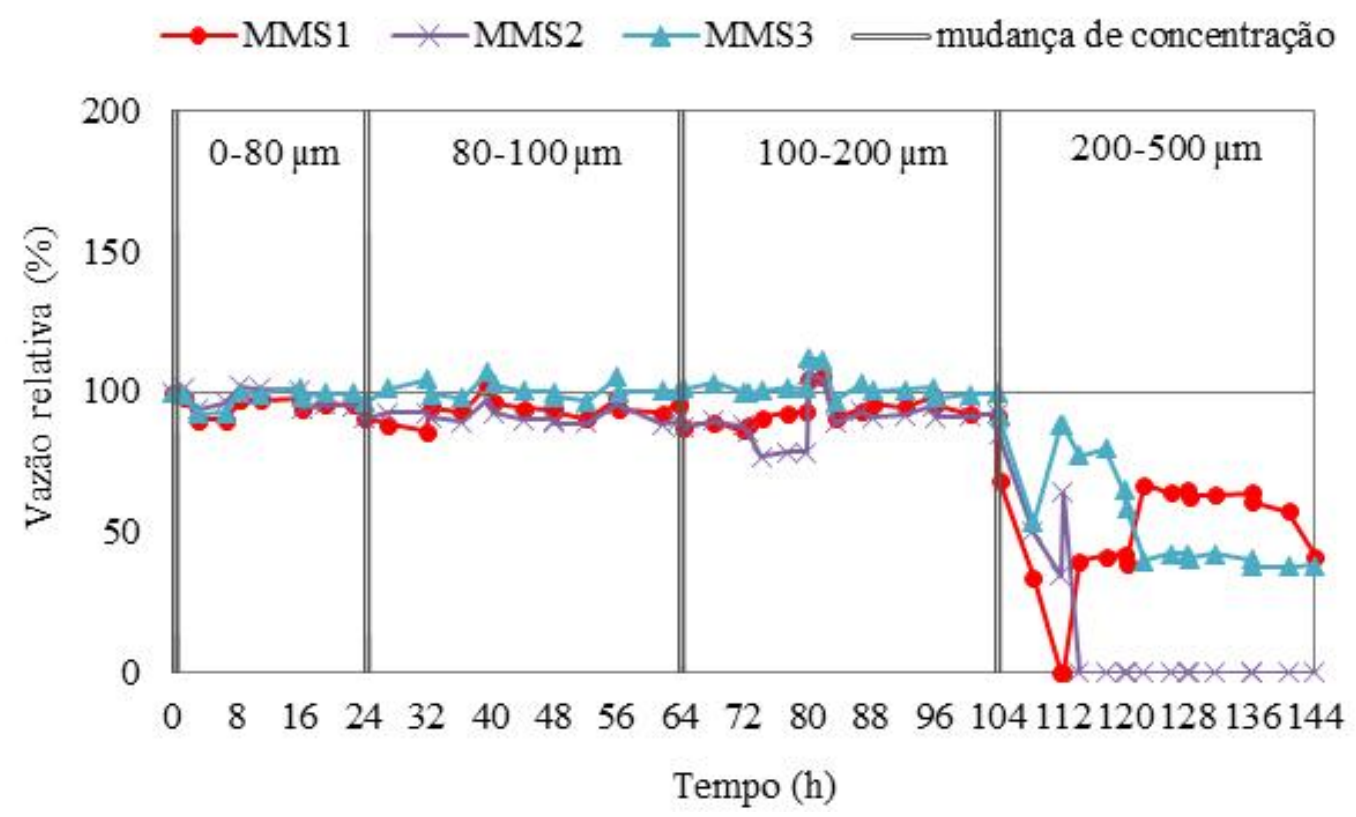

Figura 7. Vazão relativa dos microtubos com múltiplas saídas (MMS) ao longo do tempo, com o aumento de concentração e diâmetro de partículas de areia na verificação da sensibilidade à obstrução

A presença de partículas de areia e de alguns materiais não identificados em todos os conectores pode ser visualizada na Figura 8. Portanto, as partículas de areia estavam se acumulando no conector ao invés de passarem para os microtubos emissores ligados a ele (Figura 8C). Isso pode ter favorecido para que não acontecesse a obstrução dos emissores nas três primeiras etapas do ensaio. Ressalta-se que o acúmulo de partículas de areia ocorreu nas áreas com velocidade da água no conector era igual ou próxima a zero, as quais foram identificadas por meio das simulações utilizando o programa computacional ANSYS Fluent ${ }^{\circledR}$. Além disso, a obstrução ocorreu apenas na entrada do microtubo emissor que estava ligado ao conector. Nenhum dos microtubos reguladores de pressão pertencentes ao MMS estavam obstruídos. Resultados semelhantes foram verificados no trabalho de Gamri et al. (2014), em que as obstruções dos gotejadores testados ocorreram em áreas no interior do emissor com velocidade da água próxima ou igual a zero.

Apesar de ter sido o gotejador com menor sensibilidade a obstrução que os microtubos utilizados individualmente, ainda não se tem conhecimento de seu comportamento em períodos mais longos. Para Ravina et al. (1992), cada tipo de emissor possui uma sensibilidade própria ao entupimento. De acordo com os mesmos autores, emissores localizados no final da linha lateral são mais sujeitos ao entupimento do que os emissores presentes no início da linha. Porém, os primeiros emissores obstruídos neste experimento foram os que estavam mais próximos ao início da tubulação na qual os emissores estavam conectados. Isso ocorreu, provavelmente, porque o final da linha não era fechado como ocorre normalmente nos sistemas de irrigação.

Segundo Ayers e Westcot (1999), a obstrução por sólidos em suspensão, os quais acontecem com maior frequência nas águas superficiais, é o problema mais comum e também o mais fácil de solucionar, podendo-se utilizar: a sedimentação para as partículas mais pesadas - método mais antigo e barato, contudo sua eficácia não é segura; e a filtração - é mais confiável desde que o elemento filtrante seja compatível com o orifício de saída dos emissores.

Ao analisar a parte interna dos microtubos emissores, microtubos regulador de pressão e dos microtubos com múltiplas saídas não foram encontrados sinais de obstrução ao longo de seu comprimento, ou seja, a obstrução ocorria apenas na entrada dos microtubos. 

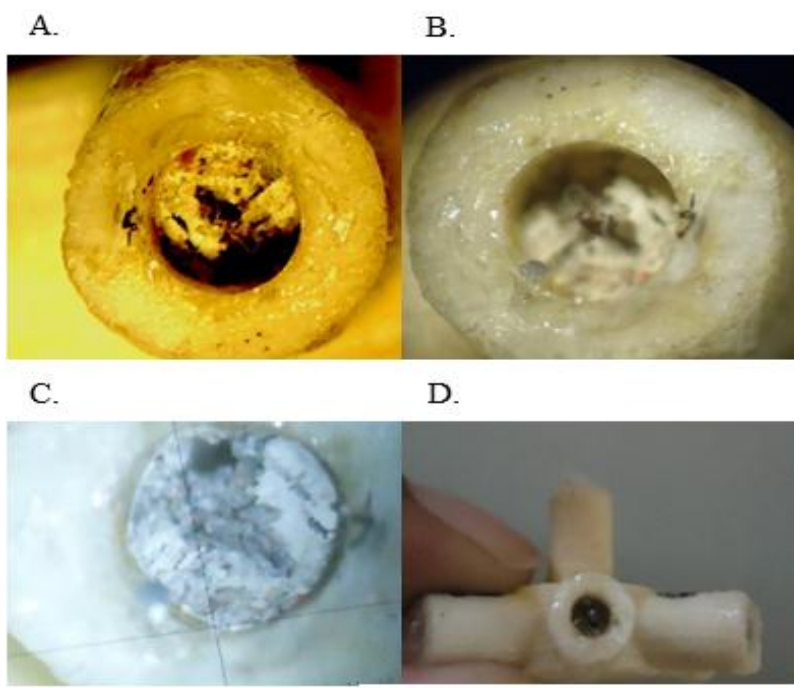

E.

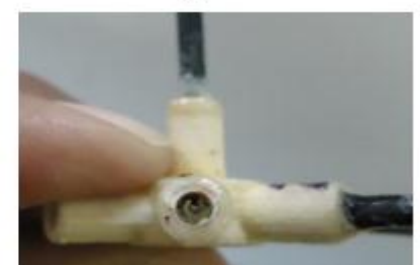

Figura 8. Detalhes dos conectores obstruídos: Imagens obtidas com microscópico eletrônico (A, B e C), imagens obtidas com câmera digital comum (D e E)

\section{CONCLUSÕES}

O microtubo com múltiplas saídas é menos sensível à obstrução que o microtubo individual com diâmetro interno de 1,074 mm;

A simulação permitiu identificar as áreas críticas no conector que podem contribuir para a obstrução do emissor e os pontos que causam a maior dissipação de energia;

As principais obstruções observadas aconteceram no interior do conector e quando o fluxo ocorre em direção ao microtubo emissor onde a velocidade da água é próxima ou igual a zero.

\section{AGRADECIMENTOS}

A Coordenação de Aperfeiçoamento de Pessoal de Nível Superior (CAPES) pela concessão da bolsa de doutorado sanduíche por meio do Programa de Doutorado Sanduíche no Exterior (PDSE) - Processo $n^{0}$ 6163-13-0. Ao Ministério da Ciência e Tecnologia (MCT), ao
Conselho Nacional de Desenvolvimento Científico e Tecnológico (CNPq) e à Fundação de Amparo à Pesquisa do Estado de São Paulo (FAPESP), pelo apoio financeiro a esta pesquisa, através do Instituto Nacional de Ciência e Tecnologia em Engenharia da Irrigação (INCTEI).

\section{REFERÊNCIAS}

ALMEIDA， C.D.G. C.; BOTREL， T.A.; SMITH, R.J. Characterization of the microtube emitters used in a novel micro-sprinkler. Irrigation Science, New York, v. 27, p. 209214, 2009.

ALMEIDA, C.D.G.C. de; SILVA, S.S.; ALBUQUERQUE FILHO, J.A.C. de; SILVA, E.F.F. Susceptibilidade ao entupimento de microtubos gotejadores sob fertirrigação. Irriga, Botucatu, v. 18, n. 3, p. 454-470, jul./set. 2013.

ALMEIDA, C.D.G.C.; BOTREL, T.A. Determinação do diâmetro de microtubos em irrigação localizada. Revista Brasileira de Ciências Agrárias, Recife, v. 5, p. 413-417, 2010.

ALVES, D.G.; PINTO, M.F.; SALVADOR, C.A.; ALMEIDA, A.C.S.; ALMEIDA, C.D.G.C. de; BOTREL, T.A. Modelagem para o dimensionamento de um sistema de microirrigação utilizando microtubos ramificados. Revista Brasileira de Engenharia Agrícola e Ambiental, Campina Grande, v. 16, n. 2, p. 125-132, fev. 2012.

ALVES, D.G. Desenvolvimento e avaliação de um sistema de irrigação com ultra baixa vazão utilizando microtubos ramificados. 2010. 86 p. Dissertação (Mestrado em Irrigação e Drenagem) - Escola Superior de Agricultura “Luiz de Queiroz”, Universidade de São Paulo, Piracicaba, 2010.

AYERS, R.S.; WESTCOT, D.W. A qualidade da água na agricultura. Tradução de R.S. Gheyi, J.F. de Medeiros, F.A.V. Damasceno. Campina 
Grande: UFPB, 1999. 153 p. (Estudos FAO: Irrigação e Drenagem, 29 revisado 1).

AZEVEDO NETTO, J.M.; FERNANDEZ, M.F.; ARAÚJO, R.; ITO, A.E. Manual de hidráulica. 8. ed. São Paulo: Edgard Blücher, 1998. 669 p.

DURAN-ROS, M.; PUIG-BARGUÉS, J.; ARBAT, G.; BARRAGÁN, J.; CARTAGENA, F. R. de. Effect of filter, emitter and location on clogging when using effluents. Agricultural and Water Management, Amsterdam, v. 96, p. 67-79, 2009.

FRIZZONE, J.A.; FREITAS, P.S.L. de; REZENDE, R.; FARIA, M.A. Microirrigação: gotejamento e microaspersão. Maringá: Eduem, 2012. 356 p.

GAMRI, S.; SORIC, A.; TOMAS, S.; MOLLE, B.; ROCHE, N. Biofilm development in microirrigation emitters for wastewater reuse. Irrigation Science, New York, v. 32, n. 1, p. 77-85, Jan. 2014.

GILBERT, R.G.; NAKAYAMA, F.S.; BUCKS, D.A.; FRENCH, O.F.; ADAMSON, K.C. Trickle irrigation: emitter clogging and flow problems. Agricultural and Water Management, Amsterdam, v. 3, p. 159-178, 1981.

INSTITUT NATIONAL DE RECHERCHE EN SCIENCES ET TECHNOLOGIES POUR L'ENVIRONNEMENT ET L'AGRICULTURE. IRSTEA. Disponível em: <http://www.irstea.fr/sites/default/files/ckfinder/ userfiles/files/procedure\%20essais\%20micro\%2 0irrigation.pdf $>$. Acesso em: 04 set. 2013.

KREIJ, C. DE; VAN DER BURG, A.M.M.; RUNIA, W.T. Drip irrigation emitter clogging in Dutch greenhouses as affected by methane and organic acids. Agricultural and Water Management, Amsterdam, v. 60, p. 73-85, 2003.

LI, J.; LI, Y.; ZHANG, H. Tomato yield and quality and emitter clogging as affected by chlorination schemes of drip irrigation systems applying sewage effluent. Journal of Integrative Agriculture, Amsterdam, v. 11, n. 10, p. 1744-1754, 2012.

PIZARRO CABELLO, F. Riegos localizados de alta frecuencia (RLAF): goteo, microaspersión, exudación. 3. ed. Madrid: Mundi-Prensa, 1996. 513 p.

PORTO, R.M. Hidráulica básica. 4. ed. São

Carlos: USP, EESC, 2006. 540 p.

PUIG-BARGUÉS, J.; ARBAT G.; ELBANA, M.; DURAN-ROS, M.; BARRAGÁN, J.; CARTAGENA, F. R. de, LAMM, F.R. Effect of flushing frequency on emitter clogging in microirrigation with effluents. Agricultural and Water Management, Amsterdam, v. 97, p. 883-891, 2010.

RAVINA, I.; PAZ, E.; SOFER, Z.; MARCU, A.; SCHISCHA, A.; SAGI, G. Control of emitter clogging in drip irrigation with reclaimed wastewater. Irrigation Science, New York, v. 13, p. 129-139, 1992.

SILVA, K.B. da; BATISTA, R.O.; SILVA, S.K.C.; DIAS, N.S.; COSTA, M.S. Efeito de distintas pressões de serviço na determinação da vazão em sistemas de irrigação por gotejamento operando com efluentes do processamento da castanha de caju. Agropecuária Científica no Semiárido, Patos, v. 8, n. 4, p. 89-96, out./dez. 2012.

SOUZA, R.O.R.M. Modelagem, desenvolvimento de software para dimensionamento, e avaliação de sistema de irrigação por gotejamento com microtubos. 2005. 100 p. Tese (Doutorado em Irrigação e Drenagem) - Escola Superior de Agricultura “Luiz de Queiroz", Universidade de São Paulo, Piracicaba, 2005.

SOUZA, R.O.R.M.; BOTREL, T.A. Modelagem para o dimensionamento de microtubos em irrigação localizada. Revista Brasileira de Engenharia Agrícola e 
Ambiental, Campina Grande, v. 8, p. 16-22, 2004.

TAJRISHY, M.A.; HILLS, D.J.; TCHOBANOGLOUS, G. Pretreatment of secondary effluent for drip irrigation. Journal of Irrigation and Drainage Engineering, New York, $\quad$ v. 120, n. 4, p. 716-731, July/Aug. 1994.

WEI, Q.; SHI, Y.; DONG, W.; LU, G.; HUANG, S. Study on hydraulic performance of drip emitters by computational fluid dynamics. Agricultural and Water Management, Amsterdam, v. 84, p. 130-136, 2006.
WEI, Q.; LU, G.; LIU, J.; SHI, Y.; DONG, W.; HUANG, S. Evaluations of emitter clogging in drip irrigation by two-phase flow simulations and laboratory experiments. Computers and Electronics in Agriculture, New York, v. 63, p. 294-303, 2008.

WU, D.; AU-LI, Y.; LIU, H.; YANG, P.; SUN, H.; LIU, Y. Simulation of the flow characteristics of a drip irrigation emitter with large eddy methods. Mathematical and Computer Modelling, Oxford, v. 58, n. 3/4, p. 497-506,

Aug.

2013. 